\title{
Three-dimensional morphometric analysis of human kidney nephron structures in health and disease.
}

\author{
Suneil Bhaskara, Michael Ferkowicz, Daria Barwinska and Tarek M. Ashkar (El-Achkar) \\ Indiana University School of Medicine, Department of Nephrology
}

Background and Hypothesis: Chronic kidney disease (CKD) is very common and affects as many as 37 million people in the United States. Diabetes and hypertension are the most common causes of CKD. The pathogenesis of CKD is not fully elucidated. Morphological changes such as glomerulosclerosis and tubular atrophy are commonly observed with advanced disease. However, it is unclear if such changes occur at earlier stages of disease, a time when therapeutic interventions are likely to have the most benefit. Measurements of glomerular, vascular and tubular dimensions have been typically derived from thin section, but a pipeline for acquiring these morphometric measurements in 3-dimentions have not been performed. Using enhanced tissue clearing, confocal 3D imaging and volumetric segmentation and analysis, we aimed at developing an approach to measure the dimensions of various structures within the human kidney. Our overarching hypothesis is that such approach could allow the detection of morphometric changes in early disease.

Experimental Design or Project Methods: Kidney tissue were used either from donor nephrectomies or stored frozen biopsies from Biobank Biopsy Cohort of Indiana (BBCl). Enhanced tissue clearing and staining for nuclei, endothelium and proximal tubules were performed. Optical sectioning and 3D Imaging was done using confocal microscopy. Volume rendering, segmentation and analysis were done using the Imaris Software (Bitplane). Various built in and customized segmentation approaches were used.

Results: Volumes spanning $200 \mu \mathrm{m}$ in thickness encompassing entire glomeruli and tubules were obtained. 3D Rendering of these volumes allowed visualization and enabled 3D segmentation. Dimensions of entire human glomeruli (maximum and minimum diameters, glomerular volumes and cellular densities), tubules and vessels were defined in control and samples with diabetic kidney disease.

Conclusion and Potential Impact: Our studies established an approach for accurate measurements of the dimensions of nephronal structures preserved in their 3D environment within tissue. We also established the feasibility of this approach in comparing changes with disease vs. control. Such methodology will open up a new line of investigations to better understand the pathogenesis of CKD, particularly at its early stages. 